\title{
Modelo de medición para la gestión inteǵral según el BSC en el Ministerio de Transporte de Colombia*
}

\section{Measurement model for integral management according to the BSC in the Ministry of Transportation of Colombia}

\author{
Isabel Cristina Rosero Pulido"* \\ Colmédica \\ Dora Rivera Vargas"** \\ Oficina Asesora de Planeación Ministerio de Transporte
}

\section{RESUMEN}

Este artículo aborda el desarrollo de un modelo de medición con base en la adaptación del Balanced ScoreCard o Cuadro de Mando Integral (Kaplan y Norton 1996) para el Ministerio de Transporte, con el propósito de articular la información de sus sistemas de gestión y como herramienta útil para su direccionamiento estratégico; la investigación se desarrolló en dos fases, enmarcando en la primera el diseño metodológico y tres de los cinco momentos de la investigación: planificación, trabajo de campo y análisis de la información recolectada; y en la segunda, el cuarto y quinto momento, con la adaptación del modelo Balanced ScoreCard y el informe final con la presentación de resultados que evidencian las bondades de tener una herramienta que permita la integralidad de información confiable y facilite la toma de decisiones.

\footnotetext{
Artículo de investigación

** Enfermera. Magíster en Calidad y Gestión Integral. Correo electrónico: isabel_rp86@hotmail.com CristinaR@colmedica.com

*** Ingeniera Industrial. Magíster en Calidad y Gestión Integral. Correo electrónico: drv335@hotmail.com
} 
Palabras clave: Ministerio de Transporte, sistema de gestión, direccionamiento estratégico, herramienta de medición.

\section{ABSTRACT}

This article addresses the development of a measurement model based in the Adaptation of the Robert Kaplan and David Norton's (1996) Balanced Scorecard for the Ministry of Transport in order to articulate the information management systems and as a useful tool for strategic management. The research was conducted in two phases, the methodological design and 3 of the 5 stages of the investigation were included in the first phase: Planning, fieldwork and analysis of collected information. The other 2 stages were included in the second phase as well as the adaptation of the Balanced Scorecard model and the final report with the presentation of results which show the benefits of having a tool that ensures information reliability and facilitate the decision making process.

\section{INTRODUCCIÓN}

La situación que se genera con la desarticulación de la información de cada uno de los sistemas implementados en el Ministerio de Transporte: Sistema de Gestión de Calidad - SGC, Ley 782 (2003), Modelo Estándar de Control Interno - MECI, Decreto 1599 (2005) y Sistema de Desarrollo Administrativo - SISTEDA, Ley 489 (1998); deriva en reprocesos por duplicidad de información generando pérdida de tiempo, desgaste administrativo e información poco confiable, amerita cuanto antes la implementación de modelos de medición para evaluar el desempeño de sistemas de gestión que tenga la entidad y que garanticen la transparencia, eficacia, eficiencia, efectividad y agilidad en la prestación de los servicios que ofrecen las diferentes entidades que lo componen.

En este contexto, el control de la gestión en las entidades públicas, debe realizarse mediante el direccionamiento estratégico como establecimiento de una guía de orientación válida para el cumplimiento de los objetivos institucionales. En el caso Colombiano, el gobierno nacional ha venido promoviendo la modernización de las entidades del sector público, para ejercer una dirección y control sistemáticos y transparentes y así lograr el cumplimiento de los principios que la Constitución señala como obligatorios para la administración pública, y de esta manera ofrecer productos (bienes o servicios) con altos índices de calidad a bajos costos, facilitando el acceso a los usuarios y partes interesadas, lo que conlleva a que se implementen herramientas que permitan realizar un adecuado seguimiento y medición que faciliten la obtención de información confiable y oportuna para mejorar el proceso de toma de decisiones. Lo que es coherente con el pensamiento de Sánchez (2001), cuando define "la administración pública como el resultado de un largo proceso histórico y actividad gubernamental para lograr la estabilidad, expansión y desarrollo de los gobiernos" (p. 51).

Desde los años 80, la gestión pública en Colombia ha adoptado normas para el control de la gestión de la calidad y el mejoramiento continuo, enfocando sus esfuerzos en la generación de resultados. Según la Guía Práctica para la Elaboración del Balanced ScoreCard - BSC o Cuadro de Mando Integral (CMI) (Alcaldía Mayor de Bogotá, 2007), son muchas las empresas tanto del sector privado como público que, por no encaminar la gestión sobre las necesidades y expectativas de los clientes, han terminado en fusiones o cierres por no lograr incursionar en un mundo competitivo. Lo anterior ha generado la necesidad de implementar sistemas de control que permitan monitorear el cumplimiento 
de las metas y objetivos eficiente y efectivamente, siendo uno de los métodos aplicados de mayor éxito el BSC o CMI. De igual manera, coincide con lo argumentado por Rodríguez y Ortiz (2002), en que la administración pública en general, demanda otro tipo de información distinta a la financiera, debido a que sus objetivos no se dirigen a la obtención de un resultado financiero sino a la prestación de una serie de servicios para el beneficio de la sociedad.

En ese sentido, la nueva condición de los mercados generada por las tendencias de apertura y globalización internacional de la economía, en la que se comparte la afirmación de Stiglitz (2006) de que los mercados que funcionan libremente no solamente no permiten alcanzar justicia social, sino que en muchos casos ni siquiera logran optimizar sus propios resultados productivos.

Cobra importancia observar el Balanced ScoreCard o Cuadro de Mando Integral, de Kaplan y Norton como un modelo de medición, que adecuado a las necesidades de la entidad, sirva como instrumento de medición y evaluación de la gestión estratégica que relaciona las cuatro perspectivas señaladas en el Decreto 2482 (2012), así: del cliente con transparencia, participación y servicio al ciudadano; financiera con gestión misional y de gobierno; de los procesos con eficiencia administrativa y formación y crecimiento con gestión del talento humano; definidas en las políticas del modelo de planeación y gestión que se aplican en las entidades públicas.

El objetivo de este artículo es presentar los resultados obtenidos en el trabajo de investigación al desarrollar un modelo que a través de la adaptación del BSC o CMI de Kaplan y Norton para el Ministerio de Transporte, integre información de cada uno de sus sistemas de gestión, como herramienta útil para su direccionamiento estratégico.

En el proyecto de investigación se buscó desarrollar un modelo que a través de la adaptación del BSC o CMI de Kaplan y Norton para el Ministerio de Transporte, integre información de cada uno de sus sistemas de gestión, como herramienta útil para su direccionamiento estratégico.

\section{METODOLOGÍA}

La investigación corresponde a un estudio de caso, mediante el cual se midieron y registraron los fenómenos involucrados en el problema identificado; el método utilizado fue deductivo hipotético, inicia con el establecimiento de la hipótesis, operacionalización de las variables relacionadas con los sistemas de gestión implementados, definición de indicadores, recolección y procesamiento de los datos, interpretación y presentación de resultados.

De igual manera, los criterios de validez y confiabilidad tienen fundamento en la información aportada por el Ministerio de Transporte y el universo y muestra representativa, conformado por los directivos de la entidad. Para tener acceso a la información se tuvieron en cuenta los siguientes cargos:

- Ministra de transporte

- Viceministros de transporte e infraestructura

- Secretaria general

- Director de transporte y tránsito

- Director de infraestructura

- Jefe de oficina asesora de planeación

- Jefe oficina de control interno

- Subdirector talento humano

- Subdirector administrativo y financiero

- Jefe oficina asesora jurídica 
La investigación se desarrolló en cinco momentos, a saber:

Primer momento: planificación de la investigación. En este primer momento se realiza la definición del problema.

Segundo momento: Trabajo de campo. En este segundo momento se elaboró el instrumento a partir de la identificación de los temas clasificados en las categorías que a continuación se describen, dando paso a la elaboración de las preguntas relacionadas y al instrumento de recolección de la información seleccionado: la entrevista.

Tercer momento: análisis de la información recolectada en las entrevistas. La información obtenida es analizada a través de una estrategia de codificación de la información en el aplicativo Excel para su clasificación, depuración, tabulación y generación de resultados de la entrevista aplicada; proceso que arrojó para cada una de las categorías (ver tabla 1).
Cuarto momento: adaptación del Modelo Balanced ScoreCard como herramienta que permita la articulación de los sistemas implementados en la entidad, alineados a sus objetivos estratégicos y que a través de los indicadores definidos, midan el cumplimiento de los factores críticos de éxito, así como el proceso de revisión de la información, verificación y validación.

Quinto momento: se realizó el análisis de la funcionalidad de la herramienta con la información comparativa de los 3 primeros trimestres en el 2014. Para continuar con la verificación de la información y validar los resultados obtenidos en el modelo, se aplicó una encuesta a 8 de los directivos encuestados en la fase inicial de la investigación con el objetivo de verificar la funcionalidad y utilidad del modelo BSC para el Ministerio de Transporte, una vez ejecutada la primera parte de la encuesta buscaba determinar la utilidad del modelo en el monitoreo y control de la gestión del Ministerio.

Figura 1. Definición de categorías.

\begin{tabular}{|l|l|l|}
\hline CATEGORÍAS & \multicolumn{1}{|c|}{ SIGNIFICADO } & \multicolumn{1}{c|}{$\begin{array}{c}\text { SIGNIFICADO EN EL MINISTERIO } \\
\text { DE TRANSPORTE }\end{array}$} \\
\hline Control & $\begin{array}{l}\text { Es un elemento del proceso administrativo que } \\
\text { incluye todas las actividades que se emprenden } \\
\text { para garantizar que las operaciones reales } \\
\text { coincidan con las operaciones planificadas. }\end{array}$ & $\begin{array}{l}\text { De acuerdo con el objetivo establecido en el artículo 1 del Decreto } \\
\text { cumplimiento de todos y cada uno de sus objetivos para verificar } \\
\text { el logro de los planes, programas y proyectos adoptados de } \\
\text { conformidad con el PND, con el fin de señalar debilidades y errores } \\
\text { para poder rectificarlos e impedir que se produzcan nuevamente, } \\
\text { tan como lo define Fayol. }\end{array}$ \\
\hline Oportunidad & $\begin{array}{l}\text { Definida como la posibilidad del cliente por } \\
\text { acceder a los servicios requeridos en el momento } \\
\text { adecuado, sin retrasos. }\end{array}$ & $\begin{array}{l}\text { En el Ministerio de Transporte se asocia al cumplimiento en la } \\
\text { entrega de la información solicitada, tanto interna como externa. }\end{array}$ \\
\hline Pertinencia & $\begin{array}{l}\text { Definiéndose pertinencia como la cualidad } \\
\text { de satisfacer un propósito respondiendo a las } \\
\text { necesidades y expectativas del entorno. }\end{array}$ & $\begin{array}{l}\text { Hace referencia a la coherencia entre la información solicitada, } \\
\text { la generada por cada sistema de gestión implementado en el } \\
\text { Ministerio y la suministrada tanto interna como externamente. }\end{array}$ \\
\hline
\end{tabular}




\begin{tabular}{|c|l|l|}
\hline CATEGORÍAS & \multicolumn{1}{|c|}{ SIGNIFICADO } & \multicolumn{1}{c|}{$\begin{array}{c}\text { SIGNIFICADO EN EL MINISTERIO } \\
\text { DE TRANSPORTE }\end{array}$} \\
\hline Transparencia & $\begin{array}{l}\text { Hace referencia a la claridad, veracidad, } \\
\text { accesibilidad, confiabilidad y flujo de la } \\
\text { información en la organización. También se } \\
\text { relaciona con los actos francos, abiertos y sinceros } \\
\text { de los miembros de la organización. }\end{array}$ & $\begin{array}{l}\text { Cada actuación del Ministerio de Transporte debe ser transparente } \\
\text { ante el gobierno nacional, partes interesadas y sociedad en general. }\end{array}$ \\
\hline Comunicación & $\begin{array}{l}\text { Definida como el proceso de transmitir } \\
\text { información y comprensión entre dos o más } \\
\text { personas, a través de diferentes canales y medios. }\end{array}$ & $\begin{array}{l}\text { La comunicación en el Ministerio de Transporte se relaciona con la } \\
\text { interacción que genera un eficaz y oportuno flujo de la información, } \\
\text { así como una positiva imagen institucional con todas las partes } \\
\text { interesadas y su realimentación. }\end{array}$ \\
\hline
\end{tabular}

Fuente: elaboración propia (octubre de 2013).

\section{RESULTADOS Y DISCUSIÓN Categoría de control:}

\section{Categorías del instrumento de medición}

La definición de estas categorías se realizó de acuerdo con la importancia de las temáticas más relevantes en la entidad, dado el flujo de información que generan los sistemas implementados y que son objeto de inquietudes por parte de los usuarios y sociedad en general.
La totalidad de entrevistados considera que el Ministerio debería tener una herramienta que integre los sistemas de gestión implementados, ya que esto permitiría articulación, oportunidad, disminución de errores y facilitaría el seguimiento. Respecto a los controles existentes en los sistemas de gestión, se observa que la utilidad de los mismos es reconocida, y a pesar

Figural. Categoría de control.

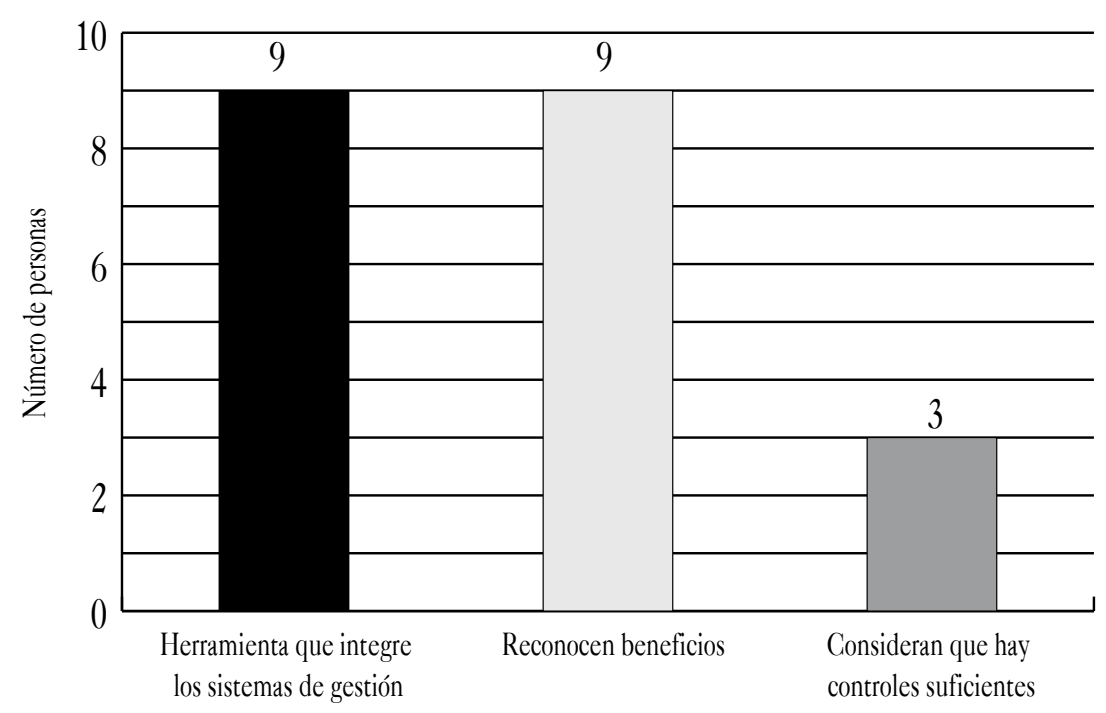

Fuente: elaboración propia (marzo de 2014). 
de ser suficientes, pueden ser modificados para mejorar la gestión de los sistemas.

En la figura 1 se muestra el resultado agrupado de la percepción que tienen los directivos del Ministerio frente al beneficio que traería incorporar la herramienta adaptada del BSC con los diferentes controles de que dispone el Ministerio de Transporte.

\section{Categoría de oportunidad:}

Se observa que la entrega de información por parte de las diferentes dependencias a los sistemas de gestión implementados en general no es oportuna, a su vez, el Ministerio de Transporte tampoco lo es con la entrega de información requerida tanto de forma interna como externa, ya que en oportunidades deben ser solicitadas prórrogas de tiempo. También se evidencia la necesidad de reforzar la realimentación sobre la información de la gestión entregada por cada dependencia con el fin de fortalecer el análisis, calidad, oportunidad y eficiencia de dicha información.

la figura 2 muestra el resultado agrupado de la percepción que tienen los directivos del Ministerio frente al beneficio que traería incorporar la herramienta adaptada del BSC, con los tiempos considerados para atender los requerimientos de información en todos en todos los ámbitos del Ministerio de Transporte.

Figura 2. Categoría de oportunidad.

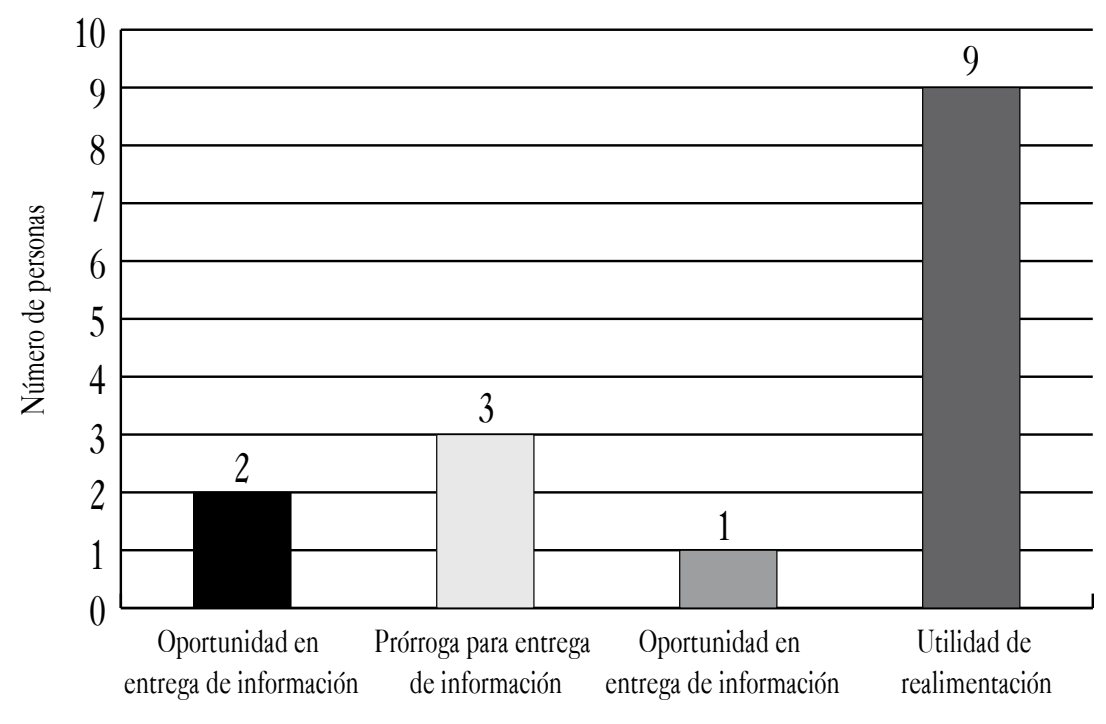

Fuente: elaboración propia (marzo de 2014).

\section{Categoría de pertinencia:}

En general, el nivel directivo tiene la sensación de que la información que generan los sistemas implementados es pertinente ya que tiene coherencia y precisión, sin embargo también se reconoce que está sujeta a ampliaciones y rectificaciones.
La figura 3 presenta el resultado agrupado de la percepción que tienen los directivos del Ministerio frente al beneficio que traería incorporar la herramienta adaptada del BSC, con información consistente para atender los requerimientos de usuarios y ciudadanía en general. 
Figura 3. Categoría de pertinencia.

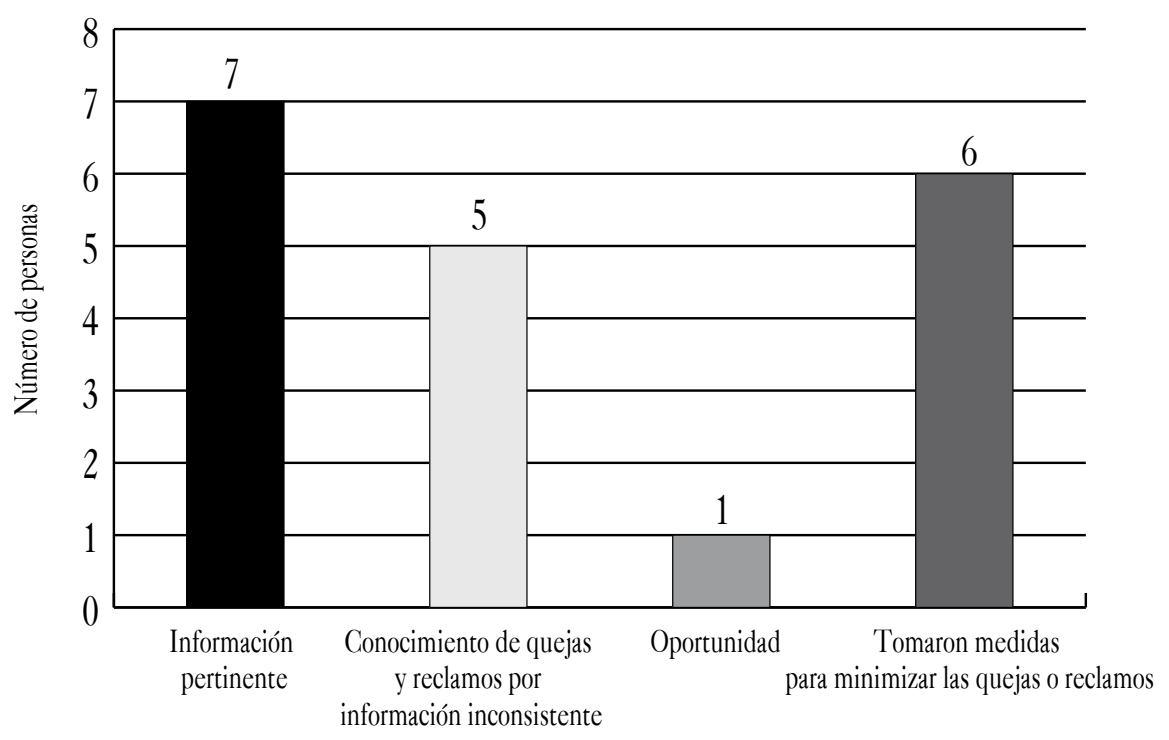

Fuente: elaboración propia (marzo de 2014).

\section{Categoría de transparencia:}

La totalidad de los entrevistados considera que el Ministerio de Transporte es transparente en sus acciones, ya que genera confianza, coherencia, sinceridad y el acceso para los usuarios es transparente y claro. Sin embargo, no se puede desconocer que eventualmente se han evidenciado focos de corrupción a través de las denuncias realizadas interna y externamente en el Ministerio, para las cuales se han tomado medidas judiciales y la aplicación de las respectivas sanciones.

En la figura 4 se presenta el resultado agrupado de la percepción que tienen los directivos del Ministerio frente al beneficio que traería incorporar la herramienta adaptada del BSC, con información consistente que

Figura 4. Categoría transparencia.

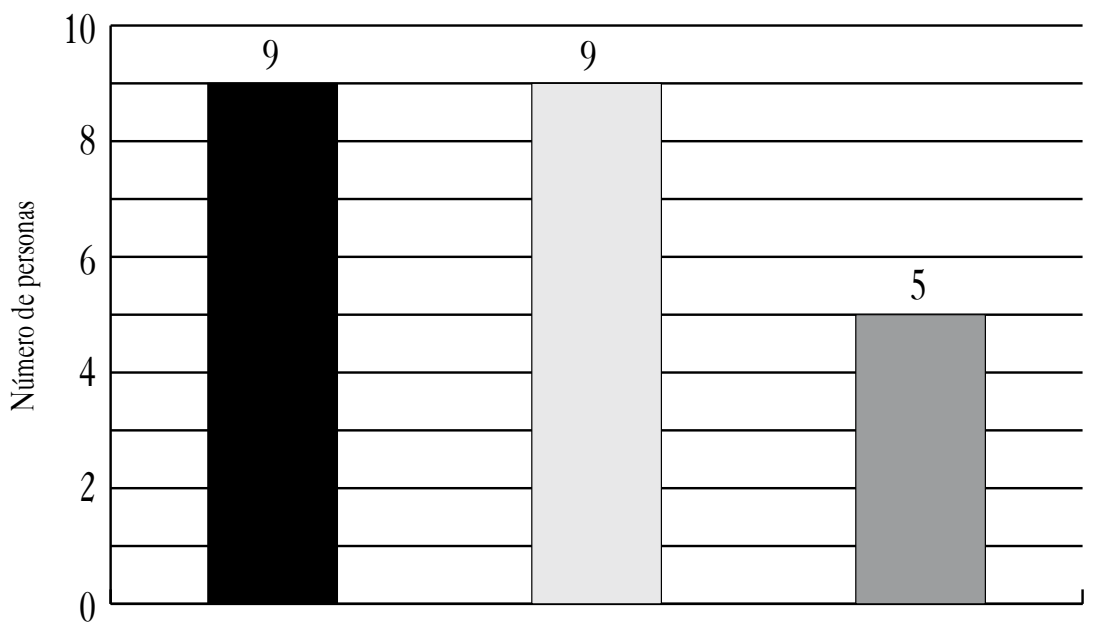

Fuente: elaboración propia (marzo de 2014). 
evidencie la actuación de la entidad, en la ejecución de los recursos asignados en el resultado de su gestión mediante el ejercicio de rendición de cuentas al gobierno nacional y ciudadanía en general.

\section{Categoría de comunicación:}

En general se observa que el Ministerio de Transporte no cuenta con una comunicación efectiva, ya que de acuerdo con la percepción de los entrevistados, no hay interacción entre los sistemas implementados, ni hay acompañamiento y los objetivos de las dependencias no están alineados con los objetivos estratégicos del Ministerio. Consideran que la comunicación proveniente de la dirección del Ministerio se debe reforzar con el fin de que sea más clara y precisa para todos los colaboradores. Con respecto al Manual de Comunicaciones, se evidencia un desconocimiento de dicha herramienta y sus directrices.

La figura 5 permite observar el resultado agrupado de la percepción que tienen los directivos del Ministerio frente al beneficio que traería incorporar la herramienta adaptada del BSC, con información consistente para atender los requerimientos de usuarios y ciudadanía en general.

Fig̉ura 5. Categoría de comunicación.

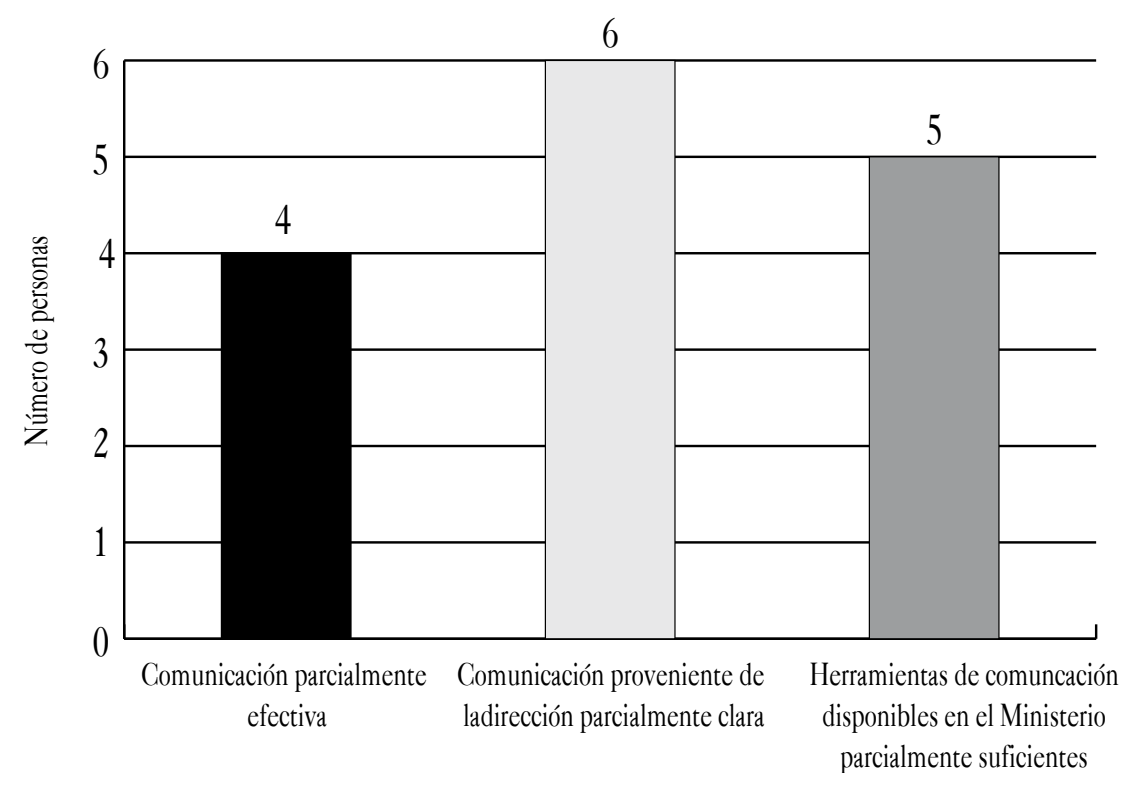

Fuente: elaboración propia (marzo de 2014).

\section{Modelo Balanced ScoreCard}

Se efectuó una adaptación del Modelo Balanced ScoreCard como herramienta que permita la articulación de los sistemas implementados en la entidad, alineados a sus objetivos estratégicos y que a través de los indicadores definidos, midan el cumplimiento de los factores críticos de éxito, así como el proceso de revisión de la información, verificación y validación. Inicialmente se establece el mapa de enlaces, para gráficamente visualizar la conectividad entre las perspectivas del modelo original con los lineamientos dados por el gobierno nacional para evaluar la gestión. 
Mapa de enlaces

Figura 6. Mapa de enlaces.

Modelo de medición para gestión integral en el Ministerio de Transporte según el BSC: estudio del caso

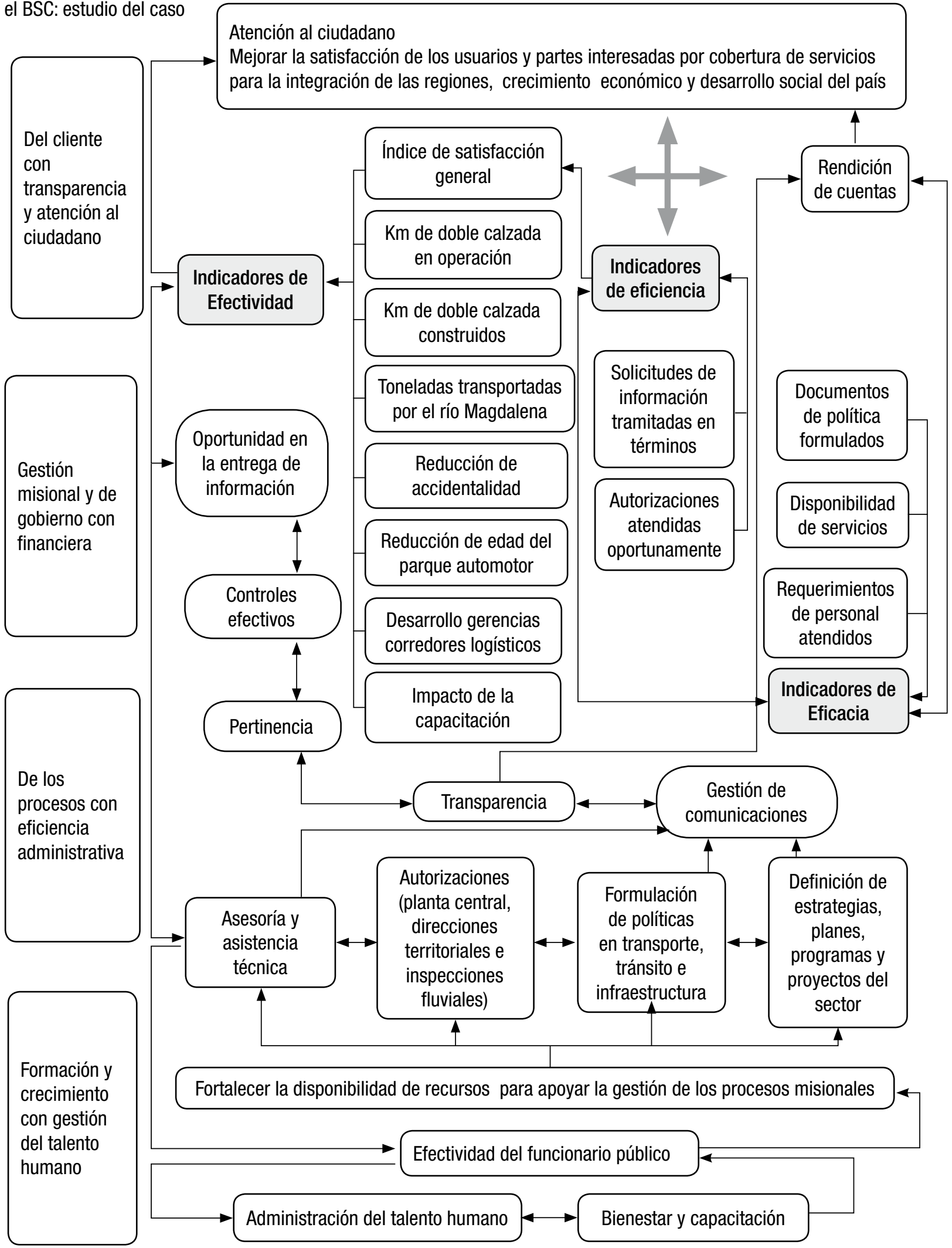

Fuente: elaboración propia (mayo de 2014). 
La figura enseña la relación que existe en la adaptación de las perspectivas del Modelo BSC, y cada una de las políticas planteadas por el Modelo de Planeación y Gestión propuesto por el Gobierno Nacional, para el logro de los objetivos estratégicos e Institucionales.

\section{Análisis de la funcionalidad de la herramienta}

De los 8 funcionarios encuestados, la totalidad considera que la implementación del modelo contribuiría en gran medida a optimizar sus procesos de gestión y control de la gestión, principalmente por la posibilidad de integrar la información generada por los sistemas de gestión ya implementados en el Ministerio de forma confiable y unificada. En cuanto al control de la gestión, los encuestados consideraron que el modelo permite establecer controles o mejorar los ya existentes para el logro de los objetivos estratégicos.

En esta figura se presenta el resultado de la verificación de la información percibida por el ámbito directivo frente a la utilidad que representa el modelo como herramienta útil en su direccionamiento estratégico.

Figura 7. Utilidad Modelo BSC.

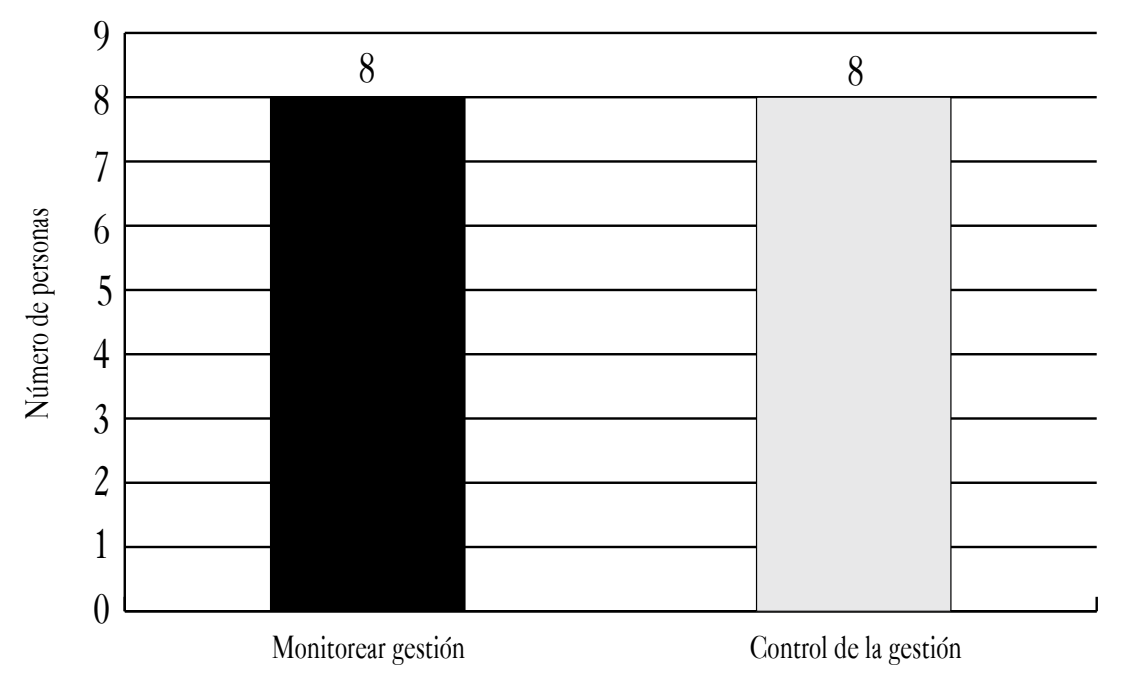

Fuente: elaboración propia (octubre de 2014).

Se indagó acerca de la pertinencia en cuanto a la selección de la información en la herramienta para el monitoreo de la gestión, encontrando que 6 de los encuestados consideran que la información integra la totalidad de los procesos, siendo de esta forma pertinente para el monitoreo de la gestión; sin embargo, 2 de los encuestados consideran que esta información se puede mejorar 0 complementar con otros aspectos para obtener una mayor certeza en los resultados arrojados por el modelo.
Esta figura muestra el resultado de la verificación de la información percibida por el ámbito directivo, frente a la utilidad del modelo en la consistencia de la información obtenida con la utilización de esta herramienta para la toma de decisiones.

Para finalizar, se verificó lo correspondiente al impacto de la aplicación de modelo en cuando a transparencia, mejoramiento de la comunicación y facilidad en 
Figura 8. Pertinencia en la información de la herramienta para el monitoreo de la gestión.

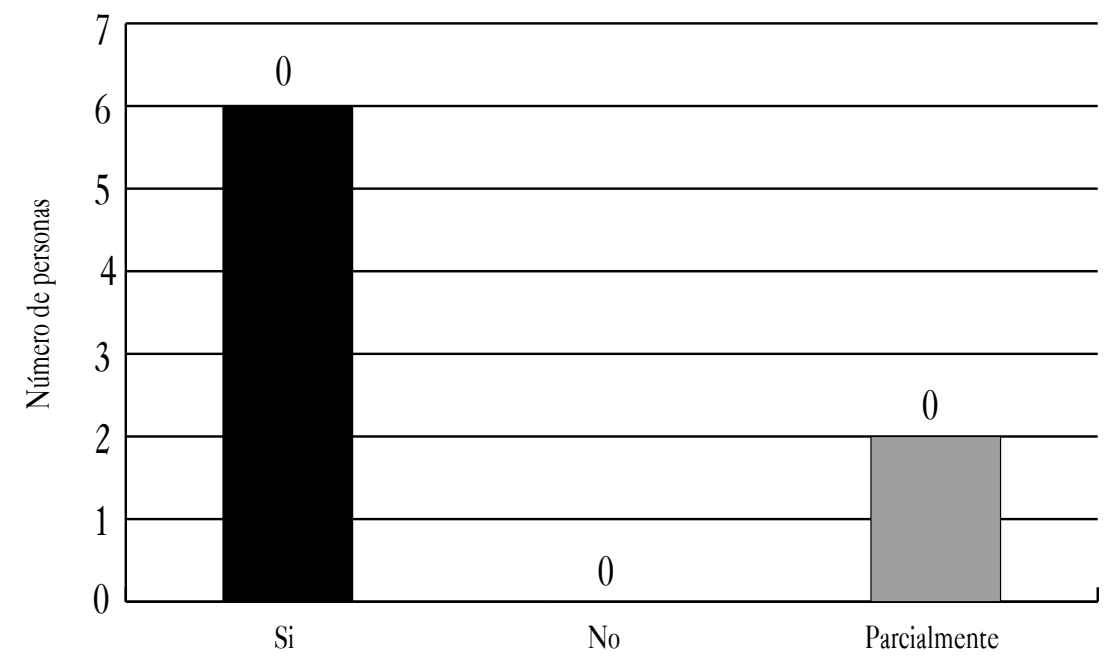

Fuente: elaboración propia (octubre de 2014).

la toma de decisiones, en donde los encuestados en su totalidad coincidieron en el aporte que realiza el modelo a dichos aspectos. En cuanto a transparencia evidencian que la calidad de la información registrada se refleja en los resultados arrojados por el modelo, siendo de esta forma evidente la importancia de la información generada por los diferentes sistemas. En el mejoramiento de la comunicación coinciden en que por la interacción de los sistemas implementados, gracias al modelo se mejora la calidad de la información y permite una comunicación fluida. Por último, la toma de decisiones mejorará porque el modelo permite ver de forma global el desempeño de la institución y permitirá la aplicación de medidas correctivas para el cumplimiento de los objetivos.

Esta figura presenta el resultado de la verificación de la información percibida por el ámbito directivo, frente a

Figura 9. Resultados obtenidos de la aplicación del modelo.

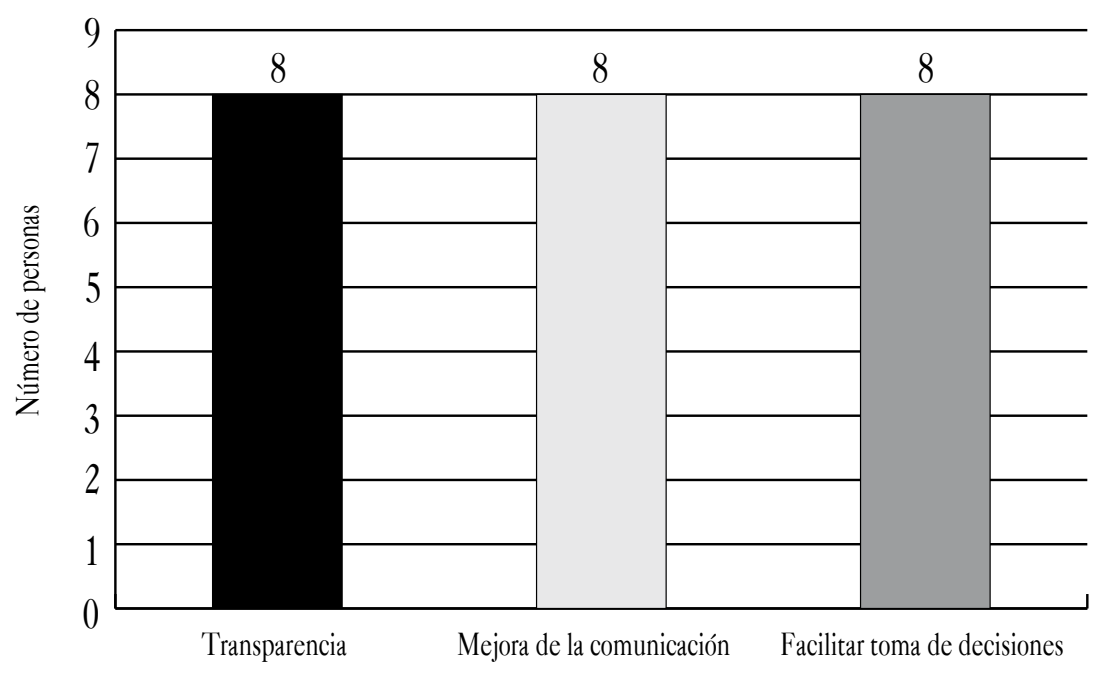

Fuente: elaboración propia (octubre de 2014). 
la utilidad que representa el modelo como herramienta útil en la obtención de información que facilita el monitoreo y control para la toma de decisiones.

\section{Análisis de resultados de la validación de la herramienta}

Una vez adaptado el modelo Balanced ScoreCard, en el aplicativo Excel, se realizó la prueba con datos estadísticos suministrados por el Grupo de Desarrollo Administrativo de la Oficina Asesora de Planeación del Ministerio de Transporte, y contenidos en el aplicativo Daruma, donde se registra la información del Sistema de Gestión de la Calidad, correspondientes al primer trimestre para contrastar la información correspondiente a cada perspectiva y la coherencia del cumplimiento de las metas de los indicadores con los porcentajes de cumplimiento frente a la calificación y color del semáforo. De la misma manera, se revisó el cumplimiento de la meta del indicador frente a las actividades definidas en cada perspectiva y su conexión con los objetivos estratégicos.

Posteriormente, se incorporó la información pertinente a cada trimestre faltante, hasta completar el cuarto trimestre e ingresar la información suministrada por el Grupo de Desarrollo Administrativo de la Oficina Asesora de Planeación, y proceder con la comparación de los resultados obtenidos en el ejercicio de los trimestres II y III de 2014 y evidenciar la coherencia con lo manifestado por los entrevistados.

Para mostrar la funcionalidad de la herramienta en la evaluación de la gestión de la entidad, a continuación se muestra cada perspectiva con sus objetivos estratégicos, actividades estratégicas, categorías, factores críticos de éxito y posteriormente los resultados obtenidos con las actividades de apoyo para el cumplimiento de las metas propuestas:

- Perspectiva cliente con transparencia, participación y servicio al ciudadano para la perspectiva del cliente con transparencia, participación y servicio al ciudadano, se tiene como objetivo estratégico y actividades estratégicas lo detallado.

Tabla 2. Objetivos estratégicos y actividades estratégicas perspectiva cliente con transparencia, participación y servicio al ciudadano.

\begin{tabular}{|c|c|}
\hline $\begin{array}{c}\text { OBJETIVOS } \\
\text { ESTRATÉGICOS }\end{array}$ & $\begin{array}{l}\text { ACTIVIDADES } \\
\text { ESTRATÉGICAS }\end{array}$ \\
\hline \multirow{3}{*}{$\begin{array}{l}\text { Mejorar los sistemas de } \\
\text { información y gestión que } \\
\text { faciliten la coordinación } \\
\text { interna, las relaciones } \\
\text { intersectoriales y la adecuada } \\
\text { divulgación a la sociedad. }\end{array}$} & $\begin{array}{l}\text { Atender las solicitudes de } \\
\text { información personalizada. }\end{array}$ \\
\hline & Atender solicitudes vía telefónica. \\
\hline & $\begin{array}{l}\text { Atender solicitudes enviadas } \\
\text { por medios electrónicos (correo } \\
\text { electrónico, chat, página web, } \\
\text { redes sociales). }\end{array}$ \\
\hline
\end{tabular}

Fuente: elaboración propia (octubre de 2014).

Este es el objetivo estratégico enfocado a la atención al ciudadano y las actividades estratégicas que facilitan la asignación de responsabilidades a los funcionarios que participan en dicho proceso, a través de los diferentes canales de comunicación con los usuarios y ciudadanía en general.

La herramienta permite hacer el seguimiento a los resultados alcanzados en cada una de estas actividades y su impacto en el logro de la gestión misional, desde el punto de vista de cada perspectiva, como se muestra en la figura comparativo periodos I, II y III de 2014 del cliente con transparencia, participación y servicio al ciudadano.

La figura compara el seguimiento al cumplimiento de metas y el resultado de la información obtenida en los tres primeros periodos de la vigencia 2014, así como la consistencia de la misma frente a los mecanismos de que dispone la entidad para medir el cumplimiento de su gestión. 
Figura 10. Comparativo periodos I, II y III de 2014 del cliente con transparencia, participación y servicio al ciudadano.

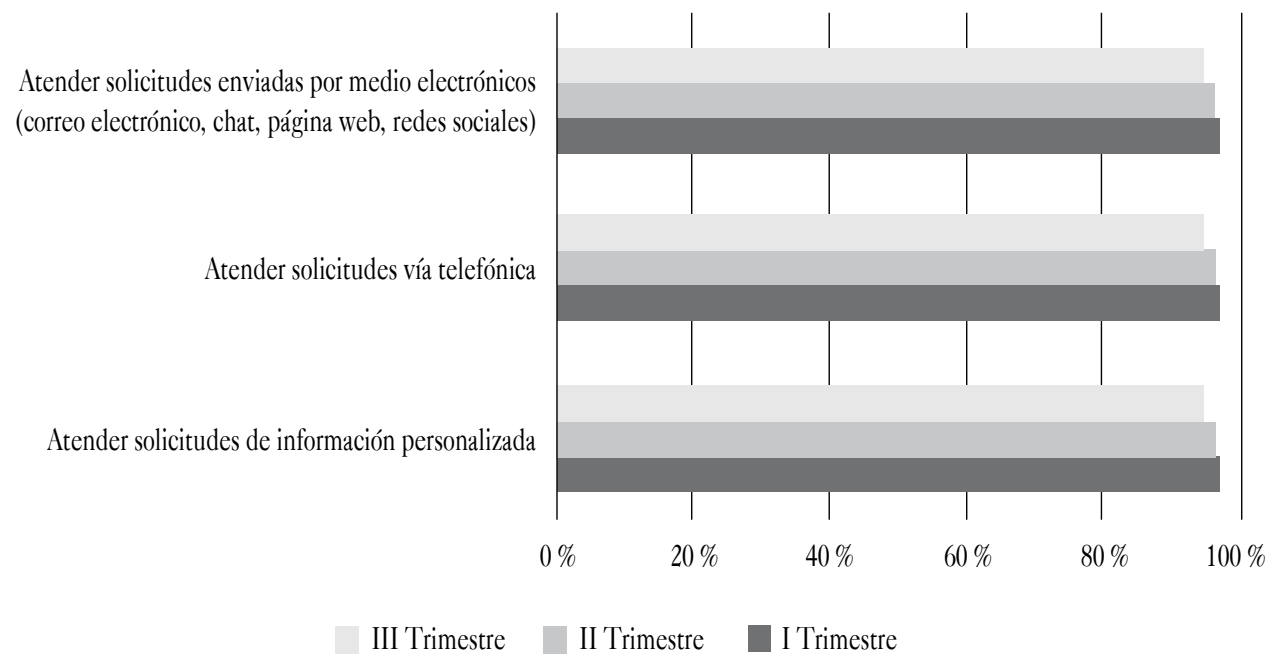

Fuente: elaboración propia (octubre de 2014).

Se observa que el modelo presenta como resultado el cumplimiento de la meta con tendencia a disminuir levemente en los periodos I y II, sin embargo, se evidencia un descenso notable en 2 puntos entre los periodos II y III; lo anterior, frente a los datos obtenidos en las fuentes de evaluación de que dispone el Ministerio, periodos en que es sometida a prueba la herramienta.

Para mejorar estos resultados se tiene previsto la revisión tanto del instrumento que es la encuesta de satisfacción, así como de su forma de aplicación para conocer la percepción de los usuarios de los servicios del Ministerio de Transporte e igualmente revisar el indicador y su forma de medición.

- Perspectiva: financiera con gestión misional y de gobierno para la perspectiva financiera con gestión misional y de gobierno, se tienen como objetivos estratégicos y actividades estratégicas los detallados en la tabla 3.

Tabla 3. Objetivos estratégicos y actividades estratégicas perspectiva financiera con gestión misional y de gobierno.

\begin{tabular}{|l|l|}
\hline \multicolumn{1}{|c|}{ OBJETIVOS ESTRATÉGICOS } & \multicolumn{1}{c|}{ ACTIVIDADES ESTRATÉGICAS } \\
\hline $\begin{array}{l}\text { Promover la inserción estratégica de Colombia en el } \\
\text { comercio internacional. }\end{array}$ & $\begin{array}{l}\text { Construcción y consolidación de corredores que soportan carga de comercio exterior } \\
\text { y que conectan los principales centros de producción y consumo con los puertos } \\
\text { marítimos, aeropuertos y pasos de frontera a través del programa dobles calzadas para la } \\
\text { prosperidad. }\end{array}$ \\
\hline $\begin{array}{l}\text { Impulsar un sistema de transporte de clase mundial, } \\
\text { integrado e intermodal. }\end{array}$ & $\begin{array}{l}\text { Realización de obras de adecuación, rehabilitación, construcción y mantenimiento } \\
\text { de muelles, encausamiento de las vías navegables, además de la instalación, } \\
\text { implementación y mantenimiento de señalización y balizaje. }\end{array}$ \\
\hline
\end{tabular}




\begin{tabular}{|l|l|}
\hline \multicolumn{1}{|c|}{ OBJETIVOS ESTRATÉGICOS } & \multicolumn{1}{c|}{ ACTIVIDADES ESTRATÉGICAS } \\
\hline $\begin{array}{l}\text { Aumentar cobertura y nivel de servicio en la } \\
\text { infraestructura de transporte. }\end{array}$ & $\begin{array}{l}\text { Promover la homogenización de la capacidad vial, así mismo, se adoptarán mecanismos } \\
\text { para asegurar su eficaz ejecución y se implementarán acciones para contar de manera } \\
\text { rápida con la infraestructura en servicio que, adicionalmente, ofrezca condiciones de } \\
\text { seguridad y durabilidad frente a situaciones de vulnerabilidad ambiental o fenómenos } \\
\text { de remoción en masa tales como deslizamientos, flujos de lodos o caída de rocas. El } \\
\text { Gobierno nacional también fortalecerá los programas de rehabilitación y mantenimiento } \\
\text { periódico y rutinario en la red vial nacional. }\end{array}$ \\
\hline $\begin{array}{l}\text { Reducir costos logísticos y tiempos de movilización. } \\
\text { Adaptar la infraestructura a condiciones de cambio } \\
\text { climático, conservación y uso adecuado de los } \\
\text { ecosistemas, apoyando la reducción de emisiones } \\
\text { contaminantes y de gases efecto invernadero en la } \\
\text { operación del transporte. }\end{array}$ & $\begin{array}{l}\text { Estructurar y poner en funcionamiento Corredores Logísticos. } \\
\text { Implementar el procedimiento de reconocimiento económico a los propietarios } \\
\text { de vehículos de carga que se acojan al proceso de desintegración física total esto } \\
\text { conlleva a la verificación de la documentación adjunta a las diferentes solicitudes de } \\
\text { postulación y posteriormente a la expedición de los actos administrativos que otorgan el } \\
\text { reconocimiento otorgado por el Estado. }\end{array}$ \\
\hline $\begin{array}{l}\text { Reducir índices de accidentalidad de tránsito, así como } \\
\text { de eventos de inseguridad. }\end{array}$ & $\begin{array}{l}\text { Implementar el Plan Nacional de Seguridad Vial a través de la implementación de } \\
\text { Planes locales, Departamentales y Regionales de Seguridad Vial, Implementación } \\
\text { yoperación del observatorio Nacional de seguridad vial y apoyo a las entidades } \\
\text { territoriales para el diseño en implementación de observatorios. }\end{array}$ \\
\hline
\end{tabular}

Fuente: elaboración propia (octubre de 2014).

Este cuadro muestra la traducción de estos objetivos estratégicos en actividades estratégicas que facilitan la asignación de responsabilidades a los funcionarios que participan en los diferentes procesos misionales tanto en la Entidad como en el Sector, realizando acompañamiento, asesoría, revisión y seguimiento a los proyectos de inversión, a través del Sistema Unificado de Inversión y Finanzas Públicas, dispuesto por el Gobierno Nacional, en el Departamento Nacional de Planeación de Colombia.

De acuerdo con esa información, la herramienta permite hacer el seguimiento a los resultados alcanzados en cada una de estas actividades y su impacto en el logro de la gestión misional, desde el punto de vista de cada perspectiva, como se muestra en la figura 11: comparativo periodos I, II y III de 2014.

Esta figura compara el seguimiento al cumplimiento de metas y el resultado de la información obtenida en los tres primeros periodos de la vigencia 2014, la cual es consistente con la generada por los mecanismos de que dispone la entidad para medir el cumplimiento de su gestión.

- En la figura, la herramienta arroja un resultado de $-269.80 \%$, comparado con los datos obtenidos por el Ministerio en su evaluación periódica, y que sirve como soporte para someter a prueba el modelo. Este valor está erróneamente interpretado dado que el indicador tal como se encuentra formulado actualmente, no muestra en realidad de lo que se pretende medir que es la disminución de la accidentalidad.

- Respecto al cumplimiento del indicador de reducción de la edad promedio del parque automotor en un $2 \%$, la herramienta arroja un resultado de $416.50 \%$; valor que comparado con los datos obtenidos por la entidad en su evaluación periódica y que sirve como soporte para someter a prueba el modelo; está muy por encima de lo presupuestado, 
Figura 11. Comparativo periodos I, II y III de 2014 financiera con gestión misional y de gobierno.

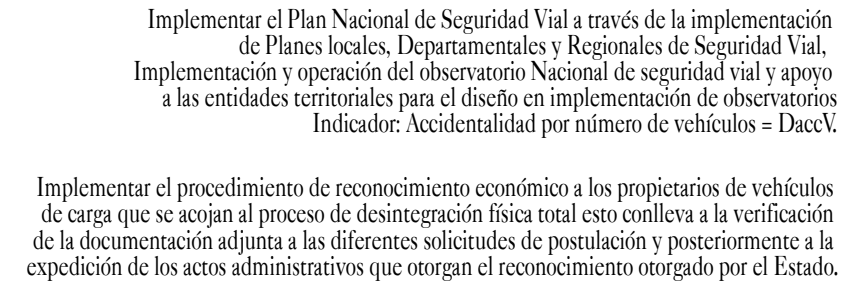

Estructurar y poner en funcionamiento Corredores Logísticos

Promover la homogenización de la capacidad vial, así mismo, se adoptarán mecanismos para asegurar su eficaz ejecución y se implementarán acciones para contar de manera rápida con la infraestructura en servicio que, adicionalmente, ofrezca condiciones de seguridad y durabilidad frente a situaciones de vulnerabilidad ambiental o fenómenos de remoción en masa tales como deslizamientos, flujos de lodos o caída de rocas. El Gobierno nacional tambien fortalecerá los programas de rehabilitación y mantenimiento periódico y rutinario en la red vial nacional

Realización de obras de adecuación, rehabilitación, construcción y mantenimiento de muelles, encausamiento de las vías navegables, además de la instalación, implementación y mantenimiento de señalización y balizaje.

Construcción y consolidación de corredores que soportan carga de comercio exterior y que conectan los principales centros de producción y consumo con los puertos marítimos, aeropuertos y pasos de frontera a través del programa Dobles Calzadas para la Prosperidad.

$$
\text { III Trimestre }
$$

Fuente: elaboración propia (octubre de 2014).

en el desarrollo de las actividades dirigidas al objetivo estratégico de adaptar la infraestructura a condiciones de cambio climático, conservación y uso adecuado de los ecosistemas, apoyando la reducción de emisiones contaminantes y de gases efecto invernadero en la operación del transporte; valor que está erróneamente interpretado dado que el indicador tal y como se encuentra formulado actualmente, no refleja en realidad de lo que se pretende medir que es la disminución de la edad del parque automotor.

- En relación con el indicador de gerencias de corredores logísticos y su meta de 1 para esta vigencia, no se cumple.

- Frente al indicador relacionado con las actividades que se desarrollan para lograr el objetivo estratégico

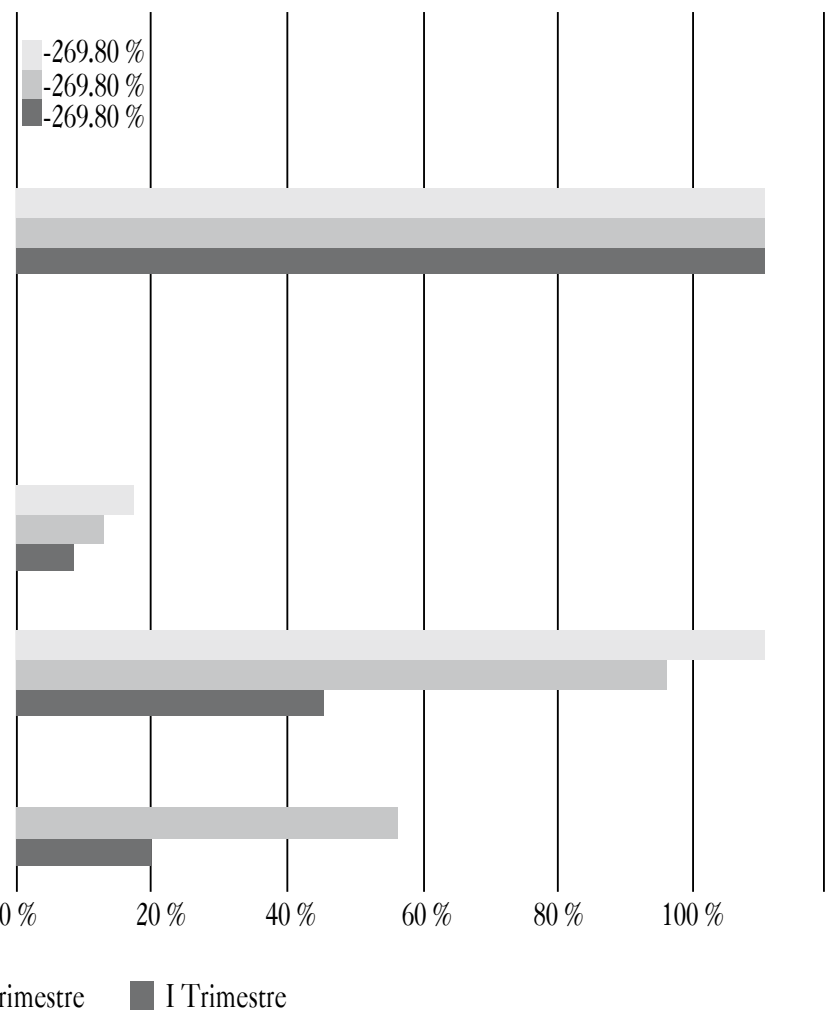

II Trimestre I Trimestre 
meta en el tercer periodo, en un $17 \%$ respecto a lo presupuestado; periodos en que fue sometida a prueba la herramienta.

- En relación con el indicador correspondiente a las actividades que se desarrollan para lograr el objetivo estratégico de promover la inserción estratégica de Colombia en el comercio internacional mediante la puesta en operación de nuevos $\mathrm{km}$ de doble calzada, se encuentra que respecto a la meta para la vigencia 2014 de 150,13 km, la herramienta presenta un resultado de 0 en cada periodo evaluado, periodos que fueros sometidos a prueba respecto a los datos proporcionados por el Ministerio y con base en los cuales realiza el seguimiento al cumplimiento respecto a la meta para la vigencia 2014.

Concuerda este análisis con lo asegurado por los usuarios de la herramienta, en cuanto al valor que agregaría el uso de esta herramienta en especial en esta perspectiva, porque encierra de manera global el desempeño de la misión institucional, el enlace de las actividades que apuntan a generar mayor impacto en el desarrollo del país, lo que permitirá la aplicación oportuna de medidas para el cumplimiento de los objetivos estratégicos.

Perspectiva: procesos con eficiencia administrativa para la perspectiva de procesos con eficiencia administrativa, se tienen objetivos y sus actividades estratégicas.

Tabla 4. Objetivos estratégicos y actividades estratégicas perspectiva procesos con eficiencia administrativa.

\begin{tabular}{|l|l|}
\hline \multicolumn{1}{|c|}{ OBJETIVOS ESTRATÉGICOS } & \multicolumn{1}{c|}{ ACTIVIDADES ESTRATÉGICAS } \\
\hline \multirow{4}{*}{$\begin{array}{l}\text { Mejorar los sistemas de información y gestión que } \\
\text { faciliten la coordinación interna, las relaciones } \\
\text { intersectoriales y la adecuada divulgación a la } \\
\text { sociedad. }\end{array}$} & $\begin{array}{l}\text { Realizar mantenimientos preventivos y correctivos a los sistemas de información y } \\
\text { comunicaciones del MT (sistema de gestión documental Orfeo, correo electrónico, } \\
\text { Sirena, Galeón, Core, Ravec, enlaces de internet e intranet). }\end{array}$ \\
\cline { 2 - 3 } & $\begin{array}{l}\text { Realizar mantenimientos preventivos y correctivos a la infraestructura tecnológica del } \\
\text { MT (servidores Blade y UPS). }\end{array}$ \\
\hline \multirow{5}{*}{$\begin{array}{l}\text { Ofrecer seguridad jurídica a los agentes que } \\
\text { intervienen en el sector a través de un marco } \\
\text { normativo y regulatorio estable y coherente. }\end{array}$} & $\begin{array}{l}\text { Brindar asesoría y asistencia técnica a los usuarios en temas de transporte, tránsito e } \\
\text { infraestructura. }\end{array}$ \\
\cline { 2 - 3 } $\begin{array}{l}\text { Atender las solicitudes de autorización presentadas por los usuarios en temas de } \\
\text { transporte, tránsito e infraestructura. }\end{array}$ \\
\cline { 2 - 2 } & $\begin{array}{l}\text { Formular políticas en transporte, tránsito e infraestructura, con base en identificación de } \\
\text { necesidades. }\end{array}$ \\
\cline { 2 - 2 } & $\begin{array}{l}\text { Defender administrativa, judicial y extrajudicialmente los intereses jurídicos y } \\
\text { económicos del Ministerio de Transporte. }\end{array}$ \\
\hline
\end{tabular}

Fuente: elaboración propia (octubre de 2014).

En esta tabla se observan los objetivos estratégicos ligados a la gestión interna de la entidad y las actividades estratégicas que facilitan la asignación de responsabilidades a todos los funcionarios que participan en cada uno de los procesos, creando una sinergia que facilita el flujo de la información que genera cada sistema implementado, para contribuir al logro de los objetivos institucionales. 
Figura 12. Comparativo periodos I, II y III de 2014, procesos con eficiencia administrativa.

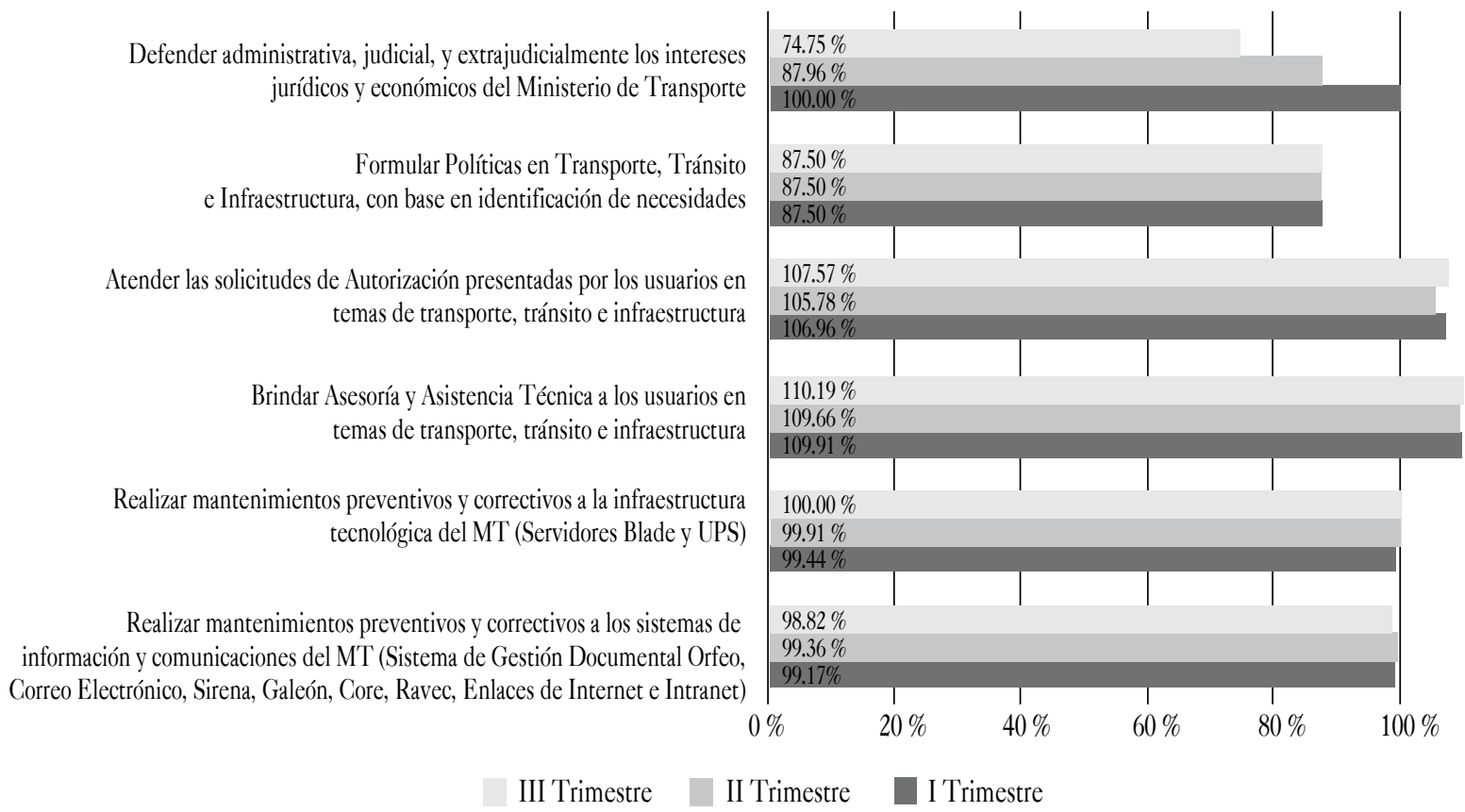

Fuente: elaboración propia (octubre de 2014).

Allí se observa la contribución de los procesos que apuntan al logro de estos objetivos estratégicos, y evidencian el cumplimiento de metas, actividades estratégicas y de apoyo en cada uno.

- El modelo presenta como resultado para el proceso de defensa judicial, cumplimiento de la meta establecida, en coincidencia con la información obtenida en la medición periódica efectuada en el aplicativo Daruma, dispuesto para ello por la entidad y probada de igual manera en la herramienta.

- Para el proceso de formulación de políticas en transporte, tránsito e infraestructura, el modelo arroja un cumplimiento del $87.5 \%$, en correspondencia con la información obtenida sobre el cumplimiento de la meta propuesta y evaluada en el aplicativo Daruma, dispuesto por la entidad y sometida a prueba con la misma periodicidad en el modelo.
- El modelo arroja resultado de cumplimiento en el proceso de autorizaciones, en contraste con los resultados obtenidos por la entidad, en el aplicativo dispuesto para ello, donde se evalúa el cumplimiento en cada periodo definido para la vigencia en análisis y que sirve como soporte para someter a prueba la herramienta, y sometida a prueba con la misma periodicidad en el modelo.

- Referente al proceso de asesoría y asistencia técnica, se observa que la meta propuesta como en el proceso de autorizaciones es del $90 \%$, el modelo da como resultado el cumplimiento de la misma y con la misma periodicidad de evaluación, comparada con los datos obtenidos y analizados por el Ministerio en el mecanismo de medición dispuesto para el cumplimiento de sus indicadores y que sirve como soporte. 
- En relación con los indicadores de mantenimiento preventivo y correctivo tanto de los sistemas de información como de disponibilidad de la plataforma tecnológica del Ministerio, el modelo presenta un resultado de cumplimiento de la meta establecida por el Ministerio para estos procesos, en comparación con los datos obtenidos en la evaluación periódica realizada en el aplicativo Daruma, evaluados en la herramienta con la misma periodicidad para su prueba.

Se debe mantener la atención como hasta ahora, para que se continúe garantizando la prestación de los servicios y la disponibilidad ininterrumpida de la plataforma tecnológica.

Coincide lo anterior con lo expresado por los entrevistados, en cuanto a que los procesos considerados en la construcción de la herramienta arrojan resultados ligados a la información clasificada en las categorías y que relacionan los factores críticos de éxito de cada perspectiva con el cumplimiento de las metas establecidas en los indicadores para la consecución de los objetivos estratégicos.
- Perspectiva: formación y crecimiento con gestión del talento humano. Para esta perspectiva se presenta el objetivo estratégico descrito en la tabla 5. Así, la herramienta permite hacer el seguimiento a los resultados alcanzados en cada una de estas actividades y su impacto en el logro de la gestión misional, desde el punto de vista de cada perspectiva, como se muestra en la figura 13.

Esta figura expone el seguimiento al cumplimiento de metas y el resultado de la información obtenida en los tres primeros periodos de la vigencia 2014, la cual es consistente con la generada por los mecanismos de que dispone la entidad para medir el cumplimiento de su gestión.

- El modelo presenta como resultado respecto a la ejecución de programas de bienestar y capacitación, que se cumple la meta dentro de lo presupuestado, frente a la información evaluada periódicamente por el Ministerio para este indicador en el aplicativo Daruma y sometida a prueba en la herramienta con la misma periodicidad.

Figura 13. Comparativo periodos I, II y III de 2014, formación y crecimiento con gestión del talento humano.

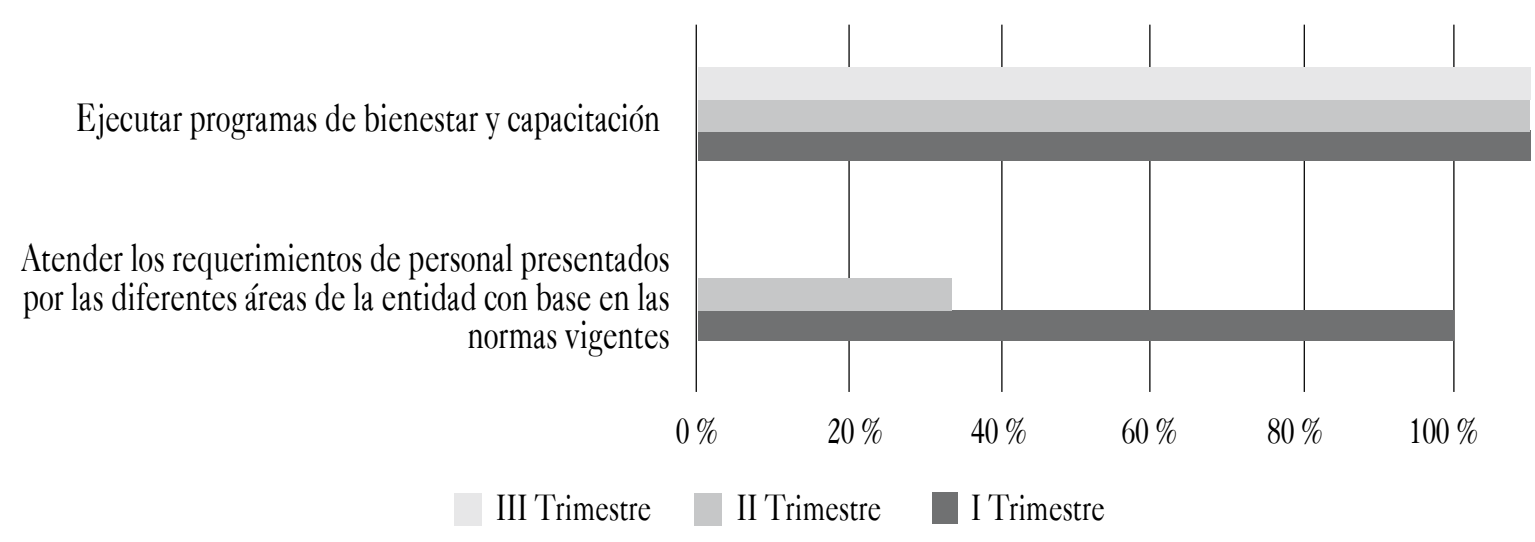

Fuente: elaboración propia (octubre de 2014). 
- En cuanto a la atención de requerimientos sobre novedades de personal, la herramienta presenta como resultado, incumplimiento total en el tercer trimestre, de la meta definida para el proceso, en concordancia con los datos por la Entidad en la medición periódica que realiza en el aplicativo Daruma y sometida a prueba en la herramienta con la misma periodicidad.

Los anteriores resultados, comprueban lo manifestado por los usuarios de la herramienta, en el sentido del aporte que tendría la incorporación de la misma en la entidad, por cuanto la información obtenida para cada perspectiva en el análisis comparativo de cada trimestre, refleja el cumplimiento de las metas, relacionadas desde los objetivos estratégicos y enlazadas a las categorías que identifican transversalmente la gestión del Ministerio, en la información de los sistemas implementados, la entidad tomará tomar acciones para corregir desviaciones y minimizar los riesgos de incumplimiento de las metas propuestas.

En general, la información obtenida como resultados en las metas para el Ministerio de Transporte, demuestra la funcionalidad del modelo, porque en cada perspectiva se observa periodo a periodo evaluado, que se mantiene dicho cumplimiento con el semáforo de color verde, advirtiendo con el de color amarillo que hay una leve variación y en el de color rojo se requiere la intervención inmediata de medidas que conduzcan a subsanar los inconvenientes que presentan algunas de sus actividades.

La articulación de la información generada por los sistemas implementados en el Ministerio de Transporte, permite a la alta dirección tener un instrumento que integre desde cada perspectiva alineada a los objetivos estratégicos con la información necesaria de cada proceso, identificado de manera transversal en las categorías que identifican la gestión de la entidad, así como con los factores críticos de éxito, para medir el cumplimiento de las metas propuestas en los indicadores en cada periodo definido, obteniendo como resultado la información presentada por el modelo frente a los datos obtenidos por el Ministerio a través de sus mecanismos de medición y en los mismos periodos evaluados, sometidos a prueba en la herramienta.

La información obtenida permitirá analizar la tomar decisiones respecto del logro de los objetivos institucionales, para revisar con cada responsable de proceso, la conveniencia o no, de replantear la forma de medir el cumplimiento de los objetivos de cada proceso, teniendo en cuenta a los funcionarios involucrados en cada uno de ellos para mantener su compromiso y contribución en el cumplimiento de la misión institucional.

\section{CONCLUSIONES}

La desarticulación de la información generada por los sistemas implementados causa un alto impacto en la gestión del Ministerio, dado que se produce información inconsistente, poco confiable, origina reprocesos y desgaste administrativo, como se pudo determinar con la aplicación de la entrevista y el análisis de la información recolectada.

La adaptación del modelo original BSC, permite evidenciar la funcionalidad de la herramienta, determinada a partir de las entrevistas de verificación de la información, lo que permite a la entidad tener una herramienta que presenta información confiable, articulada y en tiempo real de su gestión, lo que contribuirá a mejorar el proceso de toma de decisiones por la alta dirección.

La integración de los sistemas implementados en el Ministerio, cobra importancia al realizarse una interacción efectiva de información, viéndose reflejada en la adaptación de cada una de las perspectivas del modelo 
BSC con las políticas definidas para la planeación y gestión.

Los resultados que presenta el modelo, están acordes con la información tenida en cuenta para realizar los diferentes informes requeridos, tanto por las diferentes dependencias, como por los entes de control y demás partes interesadas.

El modelo presenta un sistema semaforizado de alertas, que permite visualizar a través de la ejecución de las actividades definidas en cada proceso vinculado a las perspectivas, el cumplimiento de las metas propuestas en cada trimestre.

La inclusión de la planeación estratégica así como los objetivos estratégicos y políticas básicas, se constituye en elemento fundamental para la coherencia de los resultados de la gestión frente a las directrices planteadas en el Plan Nacional de Desarrollo 2010-2014 Prosperidad para Todos.

La herramienta permite vincular en la planeación estratégica elementos importantes como la misión, visión, objetivos y actividades estratégicas, estableciendo las relaciones causa-efecto a través del mapa estratégico o de enlaces, en las cuatro perspectivas base, las cuales se adecúan según las necesidades de la entidad.

El aprovechamiento de herramientas como la adaptación del modelo Balanced ScoreCard, genera confiabilidad en la información resultante, dado que los datos ingresados en ella, son producto de la ejecución de las actividades desarrolladas en cada una de las perspectivas, durante los periodos analizados: trimestres I, II y III de 2014, en el Ministerio de Transporte.

La validación del modelo permite demostrar la consistencia de los resultados al comparar los datos obtenidos por el Ministerio en sus evaluaciones periódicas con los mecanismos dispuestos para ello, en cada una de las perspectivas y verificados estos resultados con las entrevistas efectuadas a los usuarios de la herramienta, para la toma de decisiones frente al cumplimiento de las metas establecidas por la entidad.

La coherencia de los indicadores del cuadro de mando integral propuesto, con los de los sistemas de gestión implementados en el Ministerio de Transporte, fortalecen la relación entre cada una de las perspectivas y el logro de las metas propuestas para evaluar la gestión de la entidad y así facilitar a la alta dirección la toma de decisiones.

\section{RECOMENDACIONES}

La incorporación de herramientas relacionadas con la medición y evaluación de la gestión en el Ministerio de Transporte, requiere tanto de la participación de la alta dirección porque tiene que ver con la toma de decisiones, como de los demás miembros de la entidad porque contribuyen con el desarrollo de sus actividades a la generación de información, en cada uno de los sistemas implementados y sirven como insumo para la obtención de los resultados esperados; ello confirma la necesidad de articular esta información alineada a los objetivos estratégicos, misión y visión de la entidad, por tanto se recomienda trabajar en el enriquecimiento de la herramienta para su posterior implementación.

La articulación de información que aborda los sistemas implementados en la entidad, es insumo importante a considerar en la planeación estratégica, porque permite enlazar la información a analizar y priorizar en cada uno de los procesos, esta priorización permitirá a la alta dirección evaluar el cumplimiento de las metas definidas, en consecuencia se recomienda analizar las existentes así como los indicadores.

Esta herramienta se alimenta de cada tablero de gestión de cada responsable de proceso en cada área de la 
entidad, lo que permitirá que todos los involucrados en cada proceso, entiendan la importancia de integrar sus objetivos individuales y su contribución al logro de los objetivos estratégicos, para lograr el desarrollo y ejecución de lo plasmado en el mapa de enlaces, lo que a su vez hará realidad el Plan Estratégico del Ministerio de Transporte, por lo tanto, se recomienda sensibilizar a todos los funcionarios de la entidad sobre la contribución desde su puesto de trabajo para el logro de los objetivos estratégicos.

Las autoras del estudio de investigación, con base en los resultados de las entrevistas de verificación, sugieren la necesidad de dar continuidad al proyecto por las siguientes razones:

- Incorporar en la planeación estratégica para 2015 la implementación de la herramienta en el Ministerio de Transporte, con los ajustes que se consideren pertinentes.

- Considerar los demás procesos de soporte o apoyo para enriquecer la herramienta y su contribución al logro de los objetivos institucionales.

- Tener en cuenta las nuevas disposiciones del gobierno nacional en su nuevo Plan Nacional de Desarrollo 2014-2018, en materia de objetivos y actividades estratégicos, para actualizar la herramienta.

- Hacer explícita la relación que existe entre los indicadores del modelo propuesto con los de los sistemas implementados en el Ministerio, para evidenciar la coherencia entre las mediciones de cada perspectiva y el logro de los objetivos institucionales.

\section{REFERENCIAS}

Congreso Nacional de Colombia. (1998, diciembre). Ley 489 de 1998. Por el cual se dictan normal sobre la organización y funcionamiento de las entidades del orden nacional. Colombia.

Congreso Nacional de Colombia. (2004, enero). Ley 872 de 2003. Por el cual se crea el sistema de gestión de la calidad en la Rama Ejecutiva del Poder Público y en otras entidades prestadoras de servicios. Colombia.

Congreso Nacional de Colombia. (2005, 26 de mayo). Decreto 1599. Por el cual de adopta el modelo estándar de control interno para el Estado colombiano. Diario Oficial 45920. Colombia.

Congreso Nacional de Colombia. (2012, 3 de diciembre). Decreto 2482. Por el cual se establecen los lineamientos generales para la integración de la planeación y la gestión. Diario Oficial 48634. Colombia.

Drucker, P. (1963). On the Profession of Management. Boston: Harvard Business Review Book.

Kaplan, R. S., y Norton, D. P. (1996). Balanced ScoreCard (BSC) o Cuadro de Mando Integral (CMI). Boston: Harvard Business.

Plan Nacional de Desarrollo, 2010-2014. (2011, 16 de junio). Prosperidad para Todos. Diario Oficial 48102. Colombia.

Rodríguez, M., y Ortiz, D. (2002). El cuadro de mando integral y su aplicación al control de la gestión en las administraciones públicas. España: Harvard Deusto Finanzas y Contabilidad.

Sánchez, J. J. (2001). La administración pública como ciencia: su objeto y su estudio. Recuperado de https:// 
Isabel Cristina Rosero Pulido, Dora Rivera Vargas

www.iapqroo.org.mx/website/biblioteca/LA\%20ADMINISTRACION\%20PUBLICA\%20COM0\%20 CIENCIA.pdf

Secretaria General de la Alcaldía Mayor de Bogotá. (2007, octubre). Guía práctica para la elaboración del Balanced ScoreCard (BSC) o Cuadro de Mando Integral (CMI). Bogotá: Dirección Distrital de Desarrollo Institucional.

Stiglitz, J. (2006). Cómo hacer que funcione la globalización. Buenos Aires: Taurus. 